\title{
Hierarchical Depth Mapping from Multiple Cameras
}

\author{
Jong-Il Park and Seiki Inoue \\ ATR Media Integration \& Communications Research Labs. \\ 2-2, Hikaridai, Seika-cho, Soraku-gun, Kyoto 619-02, Japan
}

\begin{abstract}
We present a method to estimate a dense and sharp depth map using multiple cameras. A key issue in obtaining sharp depth map is how to overcome the harmful influence of occlusion. Thus, we first propose an occlusion-overcoming strategy which selectively use the depth information from multiple cameras. With a simple sort and discard technique, we resolve the occlusion problem considerably at a slight sacrifice of noise tolerance. Another key issue in area-based stereo matching is the size of matching window. We propose a hierarchical scheme that attempts to acquire a sharp depth map such that edges of the depth map coincide with object boundaries on the one hand, reduce noisy estimates due to insufficient size of matching window on the other hand. We show the hierarchical method can produce a sharp and correct depth map.
\end{abstract}

\section{Introduction}

Recently, computer vision technology is widely used for achieving high degree of freedom and efficiency in video content creation. Thus, it is even said to be a kind of media technology [11].

We are developing a video component database in order to realize a flexible and versatile framework for video content creation [5]. It is based on the layered representation of video where a video sequence is regarded as a spatio-temporally ordered set of video components [17]. Video components are stored with various property information such as camera work, key words, depth, and the like in the database. We can freely select some video components from the database and enjoy arranging them in a spatio-temporal domain to make a new video and/or creating new video expressions by exploiting the given property information.

Among the information, one of the most important one would be depth. It is $Z$ value of the camera-centered coordinate of the corresponding object point for each pixel, where $Z$ axis is set to optical axis. Depth information corresponds to the spatial part in the spatio-temporal description of a scene. Thus, it takes a crucial role in making natural-looking videos and/or creating various video expressions with high degree of freedom using the video component database. Virtualized Reality [7], Z-keying for video composition [8], 3D special video effects [13], arbitrary view generation [16], and automatic scene description [14] are typical application using the depth information. Moreover, we can automatically generate multi-layer description of a scene using depth information. 
In such application, dense and sharp depth map is strongly required. Here, "sharp" means that object boundary and/or depth discontinuity should be correct. Correctness of depth map in shape is sometimes more important than precision of depth value depending on application. In this paper, how to get such a dense and sharp depth map is the main theme. The proposed method is a hierarchical scheme combined with an occlusion-overcoming disparity estimator using stereo images from 5 cameras. Considering hardware feasibility, we confine the method to a signal-level processing.

\section{Related Work}

Stereo matching is a useful method in obtaining depth map from image. However, stereo matching faces several problems such as lack of texture, occlusion, photometric change, repetitive pattern, and so on [1][2].

A considerable amount of effort has been exerted to cope with such problems in computer vision [2][10]. Almost methods to obtain dense depth map are computationally expensive or iterative. Among some exception is multiple-baseline stereo matching [12][18]. It demands more cameras but alleviates the problems of lack of texture and repetitive texture without much increase of computational complexity. Recently, a real-time depth mapper has been developed on the basis of multiple-baseline method [8].

However, little attention has been paid to clearing the occlusion problem in stereo matching. In fact, occlusion is one of the main culprits to prevent from obtaining correct depth map in shape. In two-view stereo, occlusion problem is unavoidable and it is impossible to get correct match. Only some appropriate interpolation can fill such area based on some assumption and knowledge [1]. From the standpoint of correct match, multiple view(more than two) can give a clue to resolve the occlusion problem. When an area is occluded in an image from a camera, another camera located at a different position can see the area and give a correct match. Kanade et al.'s depth mapper does not seem to explicitly exploit this property although they mentioned the occlusion problem a little [8]. Recently, Nakamura et al. extensively studied the occlusion problem [9]. Using eye array camera, they analyze occlusion patterns quantitatively and propose a disparity estimation scheme. However, it demands at least 9 cameras and furthermore, it does not provide a strategy for controlling the effect of matchingwindow size.

A very important issue underlying the area-based matching is the size of matching window [6][10]. It should be large enough to include enough intensity variation for characterizing an area. But it should be small enough to avoid projective distortion. Toward resolving such a dilemma, two approaches have been proposed. One is to use a locally adaptive window [6]. The other is to use hierarchical coarse-to-fine scheme [2][3][4][10].

In this paper, we propose a hierarchical scheme focusing on getting correct depth map around discontinuities. Two kinds of implementation are presented. One is fine-to-fine approach which requires large amount of computation [15]. 
The other is a coarse-to-fine method which alleviates computational burden considerably. We will explain the details in the following section.

\section{Depth Estimation}

\subsection{Configuration of 5 Camera System}

We put a camera at the center and a total of 4 cameras of the same specification to each direction of upper, lower, right, and left, separated by the same distance $L$. Optical axes of the 5 cameras are parallel and the cameras are synchronized. What we are to acquire is the depth map of the image from the center camera. Other cameras work as sensors in this sense.

In the configuration, the disparity $d_{t}$ of an object point is given by $d_{t}=\frac{F L}{Z}$. By estimating the disparity, we can obtain the depth of an object point.

The camera configuration is based on the assumption that when a camera cannot give a correct match for a pixel because of occlusion, another camera located at the other side can give a good one. This holds good for almost occluding cases.

\subsection{Occlusion-Overcoming Stereo Match}

We use the sum of squared-difference as a matching measure. At a point $\mathbf{x}$ on the image plane of the base camera, the matching measure is calculated at each displacement $d$ for each camera $C_{i}$ by

$$
e_{i}(\mathbf{x}, d)=\sum_{\mathbf{b} \in W}\left[I_{0}(\mathbf{x}+\mathbf{b})-I_{i}\left(\mathbf{x}+\mathbf{b}+\mathbf{d}_{i}\right)\right]^{2}
$$

where $I_{i}$ is the intensity and $W$ is a matching window.

A straightforward implementation of multiple-baseline stereo [8] would be $\hat{d}(\mathbf{x})=\arg \min _{d} \sum_{i=1}^{4} e_{i}(\mathbf{x}, d)$. It gives a good result if there is no discontinuity of depth. However, when there is a discontinuity of depth near the matching window for a pixel, we cannot expect all of the matching data from the 4 directions gives us useful information. On the contrary, some data, especially from the direction of occluded area, affect the estimation harmfully, which should be eliminated for a good estimation.

If we can eliminate such bad observations during the matching, a considerable improvement can be expected. Thus, we devise a simple sort and discard method based on the observation from $e_{i}$ curves.

We assume that at least two data sets among the four are not corrupted by occlusion. At each displacement, we sort the difference $e_{i}(\mathbf{x}, d)$ and discard the largest 2 data among the given 4 data. We sum the two data that are considered as useful for the estimation of disparity. By repeating the collection and summation of data along the epipolar line, we get a 1-D curve for the estimation and consider the displacement which gives the minimum of the curve 
as the disparity of the pixel. In short, the estimation scheme can be described by

$$
\hat{d}(\mathbf{x})=\arg \min _{d} \sum_{i=1}^{2} \tilde{e}_{i}(\mathbf{x}, d),
$$

where $\tilde{e}_{i}$ is the sorted one of $e_{i}$ such that $\tilde{e}_{i} \leq \tilde{e}_{j}$ for all $i<j$.

As we see in the Fig. 1, a considerable improvement is achieved around depth discontinuity by the above scheme at the slight sacrifice of noise tolerance as is expected [12]. The loss of noise tolerance will be compensated for by a hierarchical scheme in the following subsection. In the depth maps, we can observe two kinds of distortion. One is from occlusion. We see many noisy estimates around object boundaries in the left depth map while no such estimates in the right depth map. The other is boundary overreach. As Cochran et al. pointed out, the more strongly textured surface tends to leak into the less textured region. We see the disparity of higher texture tends to reach over the true edge of disparity and out to lower texture area in both of the depth map. The difference is that the depth map by the proposed occlusion-overcoming strategy shows clear and abrupt change around discontinuity. Moreover, the amount of the boundary overreach is roughly half of the size of the matching window [15]. This observation gives us an idea to implement a novel hierarchical scheme.
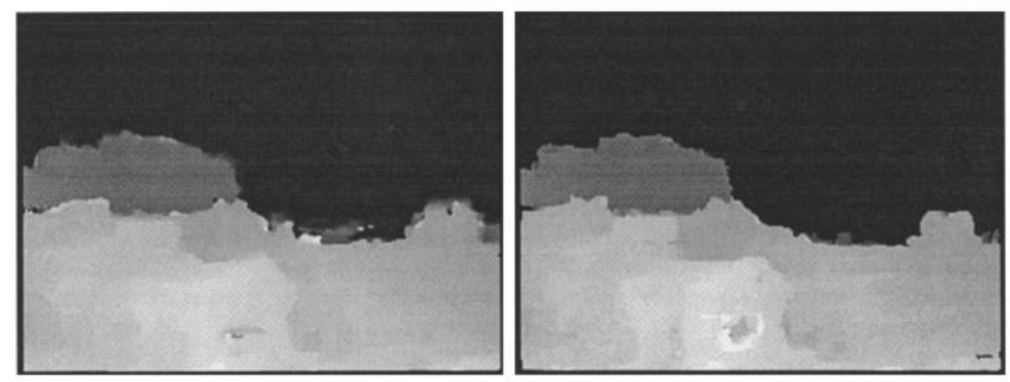

Fig. 1. Depth map by multi-camera matching. Matching window size is $15 \times 15$.

Smaller matching window is favorable in the aspect of reducing boundary overreach. But, smaller matching window gives us less reliable results of estimation. There is a trade-off between reliability and geometric correctness of estimation with respect to the size of matching window. Thus, we propose to use hierarchical schemes in order to cope with the trade-off problem as follows.

\subsection{Hierarchical Estimation Scheme}

The proposed hierarchical method is based on the observation that, when we use the occlusion-overcoming strategy, the correct disparity for boundary overreach area exists near(within half of the size of the matching window) the point in 
the disparity map in most cases. We first briefly explain the fine-to-fine implementation [15]. Then we present an efficient implementation using a resolution pyramid.

Fine-To-Fine Hierarchical Method Figure 2 illustrates the concept of the fine-to-fine hierarchical method.

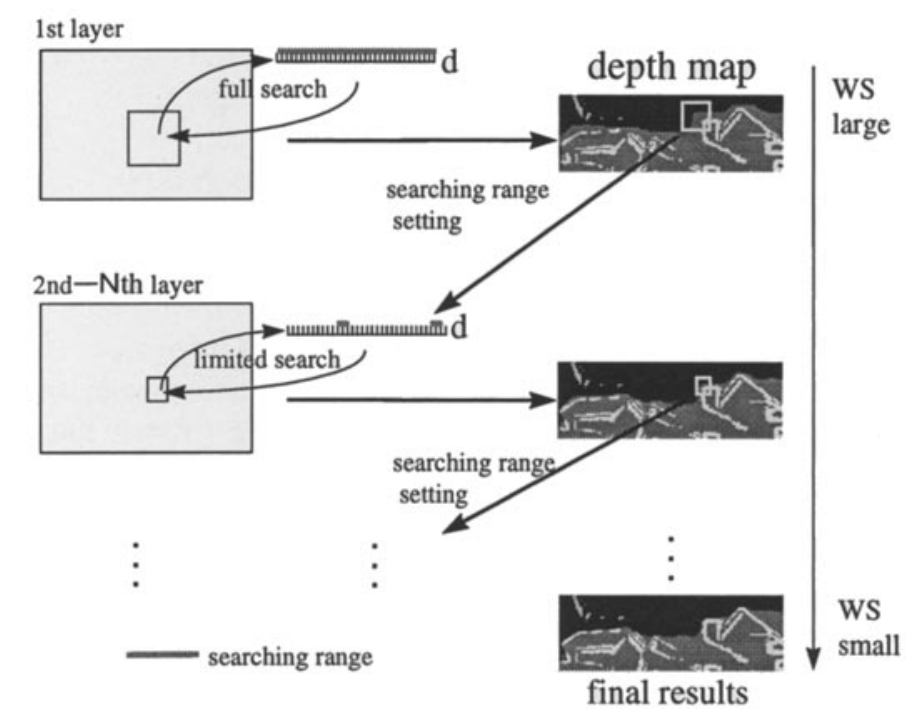

Fig. 2. Illustration of the fine-to-fine hierarchical scheme. Searching range is restricted to the estimates in the matching window of previous(=large matching window) layer except for the 1st layer.

We start by obtaining a disparity map with a large matching window using the occlusion-overcoming strategy. We assume the true disparity value of a pixel exists within the matching window of the pixel in the disparity map of the 1st layer. Now, we reduce the size of the matching window by half. Then, the disparity is estimated similarly except that the searching range is restricted to a set consisting of the disparity values of the upper layer within the window of the upper layer at the position. This restriction of searching range is based on the above observation about boundary overreach. The procedure is repeated until the last layer where the size of matching window is $3 \times 3$.

When there is no disparity discontinuity around a point(within the matching window of the point), we don't need to estimate the disparity of the point again in the successive layers. Instead, we just enhance the resolution of the disparity value to sub-pixel accuracy by quadratic fitting and no more update in the successive layers.

Coarse-to-Fine Hierarchical Method Figure 3 shows the concept of the proposed coarse-to-fine hierarchical method. 


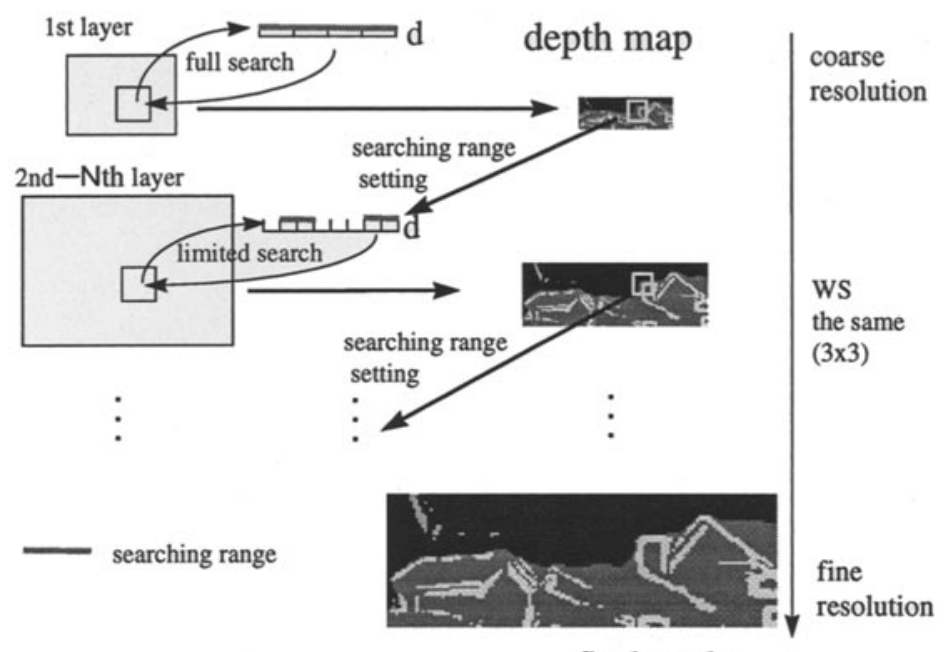

final results

Fig. 3. Mlustration of the coarse-to-fine hierarchical scheme. Searching range is restricted to the estimates in the matching window of the previous layer except for the 1st layer.

A resolution pyramid is first constructed by low-pass filtering and decimation. We use a 4-layer pyramid. The size of matching window is set to $3 \times 3$ [pixels] through the layers. Searching is performed to a pixel accuracy at each layer.

We start from the most coarse layer. Using the occlusion-overcoming strategy, we acquire the disparity map of the first layer. One pixel distance at this layer corresponds to 8 pixels at the finest layer. Thus, the disparity map is coarse in both spatial resolution and accuracy. The amount of computation is thus reduced substantially. Now we move to the 2 nd layer. The spatial resolution and the accuracy are twice those of the previous layer. We repeat stereo matching by the occlusion-overcoming strategy. But the searching range is restricted to a set consisting of the estimates in the matching window at the corresponding position of the previous layer and their neighboring disparities(See Fig.3). We repeat the same processing as of the 2 nd layer for the successive layers.

In Fig.4, we show the disparity map obtained by the hierarchical method. The fine-to-fine result is obtained by using 3 -layer $(15 \times 15$ to $7 \times 7$ to $3 \times 3)$ hierarchy. We see very clear boundaries in both of the depth maps. The coarse-to-fine result is slightly inferior to the fine-to-fine one, but not substantially.

\section{Concluding Remarks}

In this paper, we have investigated on obtaining a sharp and dense depth map from multiple cameras. The method is based on a simple discard and summation of disparity information from 5 cameras implemented on a hierarchical structure. It is confirmed that the method achieves shape-correctness of depth map. 

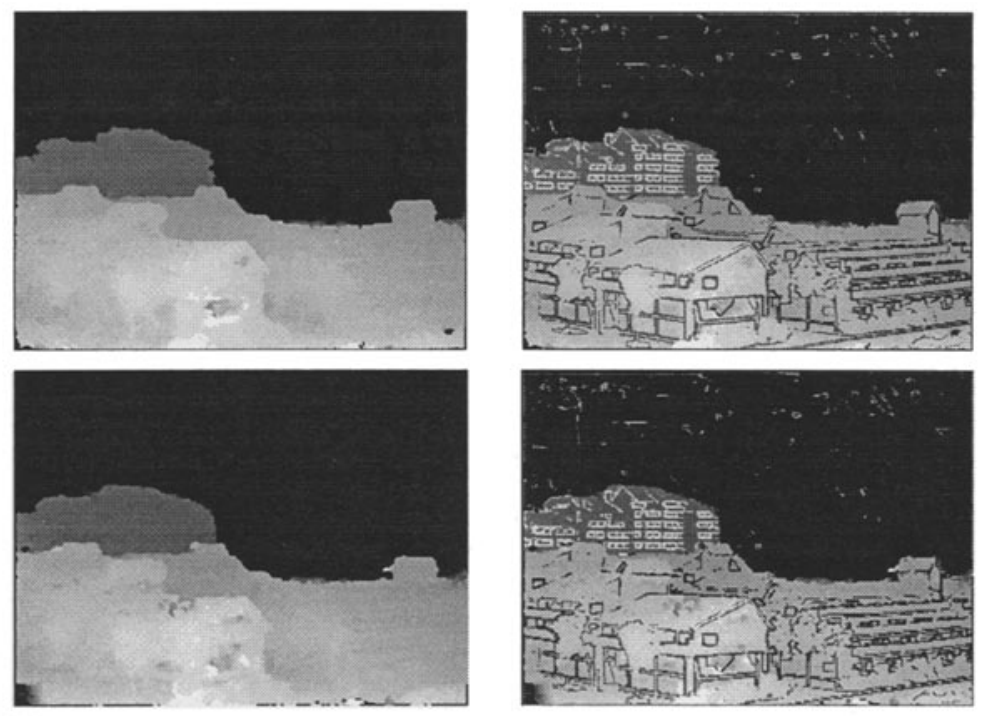

Fig. 4. Depth map obtained by the fine-to-fine [upper] and the coarse-to-fine [lower] hierarchical methods. Edge map is overwritten in the right maps.

We have tested the method over a wide variety of real images under various camera setup and confirmed the validity of the method. It is observed that the performance depends on noise level, scene complexity, and accuracy of camera calibration. The method sometimes fail to give a correct match for strongly concave areas, small holes, and narrow valley as can be reasonably predicted from the configuration of the camera system. The limitation does not seem to be resolved within the scope of non-iterative signal-level processing. Some highlevel recognition and/or interactive scheme would be necessary for overcoming this limitation.

Currently, we are developing application techniques using the depth map obtained, which include arbitrary view generation for image-based rendering, Z-key method for $3 \mathrm{D}$ video composition, automatic multi-layer description of a scene.

\section{References}

1. S.D.Cochran and G.Medioni, "3-D surface description from binocular stereo," IEEE Trans. PAMI, vol.14, no.10, pp.981-994, Oct. 1992.

2. U.Dhond and J.Aggarwal, "Structure from stereo: A review," IEEE Trans. System, Man, and Cybernetics, vol.19, no.6, pp.1489-1510, Nov./Dec. 1989.

3. W.E.L.Grimson, "A computer implementation of a theory of human stereo vision," Phil. Trans. Royal Soc. London, vol.B292, pp.217-253, 1981. 
4. M.J.Hannah, "Bootstrap stereo," Proc. ARPA Image Understanding Workshop, pp.201-208, College Park, MD, Apr. 1980.

5. S.Inoue, "Mental image expression by media integration - COMICS," Proc. of 1st International Workshop on New Video Media Technology, pp.47-52, Seoul, Korea, March 1996.

6. T.Kanade and M.Okutomi, "A stereo matching algorithm with an adaptive window: Theory and experiment," IEEE Trans. PAMI, vlo.16, no.9, pp.920-932, Sept. 1994.

7. T.Kanade et al., "Virtualized Reality: Concepts and early results," Proc. IEEE Workshop on Representation of Visual Scenes, pp.69-76, June 1995.

8. T.Kanade et al., "A stereo machine for video-rate dense depth mapping and its new applications," Proc. IEEE CVPR'96, pp.196-202, San Francisco, June 1996.

9. Y.Nakamura et al., "Occlusion detectable stereo - Occlusion patterns in camera matrix," Proc. IEEE CVPR'96, pp.371-378, San Francisco, June 1996.

10. V.S.Nalwa, A Guided Tour of Computer Vision, Addison-Wesley, 1993.

11. Y.Ohta, "Computer vision as media technology," Proc. Image Sensing Symposium, pp.265-270, 1996 (in Japanese).

12. M.Okutomi and T.Kanade, "A multiple-baseline stereo," IEEE Trans. PAMI, vol.15, no.4, pp.353-363, April 1993.

13. J.Park et al., "Extraction of depth information for scene description and its application," ITE'96, pp.112-113, Nagoya, Japan, July 1996 (in Japanese).

14. J.Park and S.Inoue, "Image expression based on disparity estimation from multiple cameras," Proc. 3rd Joint Workshop on Multimedia Communications, 7-1, Taegu, Korea, Oct. 1996.

15. J.Park and S.Inoue, "Toward occlusion-free depth estimation for video production," Proc. International Workshop on New Video Media Technology '97, 6-2, pp.131-136, Tokyo, Japan, Jan. 1996.

16. J.Park and S.Inoue, "New view generation from multi-view image sequence," Technical Report of IEICE, Sapporo, Japan, Feb. 1997 (in Japanese).

17. M.Shibata et al., "Scene describing method for video production," ITEJ Tech. Report, vol.16, no.10, pp.19-24, Jan. 1992 (in Japanese).

18. R.Tsai, "Multiframe image point matching and 3-D surface reconstruction," IEEE Trans. PAMI, vol.5, no.2, pp.159-174, March 1983. 